\title{
Epidemiological study of Appendageal skin tumors in tertiary health centre in central India
}

\author{
Rathoriya SG ${ }^{1}$, Soni SSL ${ }^{2}$, Sinha $\mathbf{U}^{3}$, Chanchlani $\mathbf{R}^{4}$ \\ ${ }^{1}$ Dr Shyam Govind Rathoriya, Assistant professor, Department of Dermatology, ${ }^{2}$ Dr Sumit SL Soni, Senior Resident, Department \\ of Dermatology, ${ }^{3}$ Dr Umesh Sinha Associate professor, Department of Community Medicine, ${ }^{4}$ Dr Roshan Chanchlani, Associate \\ Professor, Department of Surgery. All are affiliated with Chirayu Medical College \& Hospital, Bhopal, India
}

Address for correspondence: Dr. Shyam Rathoriya, Email: drshyamgr@gmail.com

\begin{abstract}
Introduction: Cutaneous appendageal tumors are a large diverse group of tumors that are commonly classified according to their state of appendageal differentiation- eccrine, apocrine, follicular and sebaceous. Most appendageal tumors are relatively uncommonly encountered in routine clinical practice. Though some of the appendageal tumors (e.g. syringoma, nevus sebaceous) can be diagnosed clinically with ease but most of them have non-specific morphological appearance and their diagnosis is mainly based on histopathological characteristics. Material and methods: It was a cross sectional descriptive study conducted in the department of dermatology, Chirayu medical college and hospital, Bhopal. A total of 90 patients attending department of dermatology were screened out for appendageal tumors and those (60 patients/66.6\%) who were confirmed by histopathological examination of lesional biopsy were included in the study. Results: On the basis of differentiation of tumors, sweat gland tumors were the most common appendageal tumors in our study (79.8\%) followed by follicular tumors $(11.6 \%)$, sebaceous were $8.3 \%$. On individual tumor basis, syringoma constituted maximum numbers of cases $(56.6 \%)$ followed by multiple trichoepitheliomas and hidrocystoma (both 11.6\%). Face was the commonest site of predilection 40(66.6\%) followed by scalp 12(20\%). Conclusion: Appendageal tumors are relatively uncommon and constitute very small proportion of dermatological disorders. Though clinical appearance might help in diagnosing common appendageal tumors but histological examination remains the gold standard method in diagnosing clinically suspicious lesions.
\end{abstract}

Key words: Appendageal tumors, Syringoma, Trichoepitheliomas

\section{Introduction}

Cutaneous appendageal tumors are a large diverse group of tumors that are commonly classified according to their state of appendageal differentiation- eccrine, apocrine, follicular and sebaceous [1]. Appendageal tumors (ATs) are neoplasms which differentiate toward or arise from pilosebaceous apparatus, apocrine gland or eccrine sweat gland.

Head \& neck region is unique because of its rich distribution of pilosebaceous apparatus, apocrine as well as eccrine sweat glands. It has also been previously Clinical diagnosis of different entity is often difficult, as most of the ATs present as asymptomatic papules or nodules. Anatomic location, number and distribution of documented that ATs predominate over head-neck area [2].

Manuscript received: $24^{\text {th }}$ Jan 2014

Reviewed: $29^{\text {th }}$ Jan 2014

Author Corrected: $15^{\text {th }}$ Feb 2014

Accepted for Publication: $28^{\text {th }}$ Feb 2014 lesions provide important clue but histopathology is invaluable in confirmation of the diagnosis [3].

Few clinicopathological studies are available on ATs from India and the world as well. Most appendageal tumors are relatively uncommonly encountered in routine clinical practice. However, diagnosing some of these tumors has important implications, as they might be markers for syndromes associated with internal malignancies, such as Cowden disease and Muir-Torre syndrome.

Though some of the appendageal tumors (e.g. syringoma, nevus sebaceous) can be diagnosed clinically with ease but most of them have non-specific morphological appearance and their diagnosis is mainly based on histopathological characteristics. The common dermatological conditions which mimic appendageal tumors are milia, basal cell epithelioma, epidermal cyst etc. 
The histopathological interpretations are mainstay for diagnosing skin ATs on biopsy. Given the importance of such uncommon conditions, this study aims at finding the frequency of ATs among all dermatology OPD patients in a tertiary care centre along with clinico-histopathological correlation in those cases.

\section{Materials and methods}

Study design: A cross sectional descriptive study was conducted over the duration of 2 yrs.

Sample size: A total of 90 patients attending department of dermatology were screened out for appendageal tumors and those (60 patients/66.6\%) who were confirmed by histopathological examination of lesional biopsy were included in the study.

Study duration: Two years (from February 2012 to January 2014).

Study setting: Department of Dermatology, Chirayu medical college and hospital, Bhopal.
Inclusion criteria: Only Biopsy confirmed cases of appendageal tumors were taken for study, other clinically diagnosed but histopathologically inconspicuous cases were excluded from the study.

Ethical clearance: Permission was taken from Institutional ethical committee.

Data analysis: Data were entered and analyzed using Microsoft excel 2007.

Procedure: The tumors were classified as eccrine, hair, sebaceous, and apocrine after a detailed histopathological examination (H/E sections).

An incidence of different tumors, their relative frequency, age \& sex predilection, nature and course, chronicity and outcome were recorded in 2 years regular follow up.

We also tried to find out the basic cause of seeking treatment, whether aesthetic or medical.

\section{Results}

60 patients $(66.6 \%)$ were confirmed after histopathological analysis which showed the high index of positivity on clinical background. Rest of the patients had appendageal/epidermal cysts and mimicked very closely to the appendageal tumors.

Out of 60 patients, enrolled for study, 24 patients were males and 36 were females with male-female ratio of approximately 1:1.5. The age group 31-40 years constituted highest numbers of patients(41.0\%) followed by $21-30$ years age group ( $34.6 \%$ ). The mean age of the subjects was $34 \pm 7.8$ years.

On individual tumor basis, syringoma constituted maximum numbers of cases $(56.6 \%)$ followed by multiple trichoepitheliomas and hidrocystoma (both 11.6\%). The distribution of the individual tumor types and frequency is given in Table No. 1

Table No 1: Distribution of the appendageal tumors according to the tumor type and frequency in patients.

\begin{tabular}{|l|l|l|l|l|}
\hline Skin Tumors & Male & Female & No. of patients & Percentage (\%) \\
\hline Syringoma & 14 & 20 & 34 & 56.6 \\
\hline Multiple Trichoepitheliomas & 3 & 4 & 7 & 11.6 \\
\hline Cylindroma & 2 & 3 & 5 & 8.3 \\
\hline Hidrocystoma & 2 & 5 & 7 & 11.6 \\
\hline Eccrine Spiradenoma & 1 & 1 & 2 & 3.3 \\
\hline Nevus Sebaceous & 2 & 3 & 5 & 8.3 \\
\hline Total & 24 & 36 & 60 & 100 \\
\hline
\end{tabular}

On the basis of differentiation of tumors, sweat gland tumors were the most common appendageal tumors in our study (79.8\%) followed by follicular tumors $(11.6 \%)$, sebaceous were $8.3 \%$. 
Table No 2: Site wise distribution of the appendageal tumors

\begin{tabular}{|l|l|l|}
\hline Site & Number of tumors & Percentage (\%) \\
\hline Face & 40 & 66.6 \\
\hline Neck & 03 & 5 \\
\hline Trunk & 00 & 0 \\
\hline Skull & 12 & 20 \\
\hline Other parts & 05 & 8.3 \\
\hline Total & 60 & 100 \\
\hline
\end{tabular}

Face was the commonest site of predilection 40(66.6\%) followed by scalp 12(20\%). Regarding site of involvement of individual tumor type, we noticed all cases of trichoepithelioma around nose and surrounding area. The commonest site for syringoma was found to be infraorbital region and cheeks. Site wise distribution of tumors is given in Table No 2.

Table No 3: Distribution of the appendageal tumors according to the duration of illness

\begin{tabular}{|l|l|l|}
\hline Duration of illness & Number of tumors & Percentage (\%) \\
\hline $0-3$ months & 6 & 10 \\
\hline $3-6$ months & 3 & 5 \\
\hline $6-12$ months & 5 & 8.3 \\
\hline $1-2$ years & 9 & 15 \\
\hline $2-5$ years & 11 & 18.3 \\
\hline $5-10$ years & 16 & 26.6 \\
\hline$>10$ years & 10 & 16.6 \\
\hline Total & 60 & 100 \\
\hline
\end{tabular}

The mean duration of illness was 7.78 years; the shortest duration being 2 months and the longest duration being 13 years. Distribution of the tumors according to the duration is shown in table No 3.

\section{Discussion}

The appendageal tumors constituted $0.46 \%$ of total patients attending skin OPD in 2 years, indicating their relatively uncommon occurrence.

Most of the patients presented to us were not even aware of the disease because of their asymptomatic \& benign nature. Majority of the appendageal tumors were diagnosed after long duration of their presence ( $57 \mathrm{pts}>10 \mathrm{yrs}$ ), yet there was hardly any effect on prognosis. In rest of the patients, the main concern of coming and seeking treatment was cosmetic disfigurement as the facial involvement was most frequent.

Syringoma (Figure No 1) was the commonest appendageal tumor (34 patients/56.6 \%) seen in this study and was characterized clinically by multiple skin coloured papular lesions, present around eyes and over cheeks and histologically by the presence of cords of epithelial cells.

Male to female ratio was 1:1.42, so overall female preponderance of appendageal tumors was found in our study, whereas in an another study done by Saha A et al in eastern India (2011), syringoma was observed in $40 \%$ of the study subjects [4].

Trichoepithelioma (Figure No 2) (7 patients /11.6\%) was seen to be the commonest follicular tumor as well as commonest appendageal tumor concentrated around nose in our study.

All the cases in this study were of the multiple types (autosomal dominant) in contrast to previous studies which showed predominance of solitary type. The tumor was characterized histologically by variably sized tumor masses with cribriform appearance and horn cyst, whereas in a study done by Samaila M in Nigeria in 2008, trichoepithelioma was seen in 3(5.8\%) of the total study subjects [5]. 


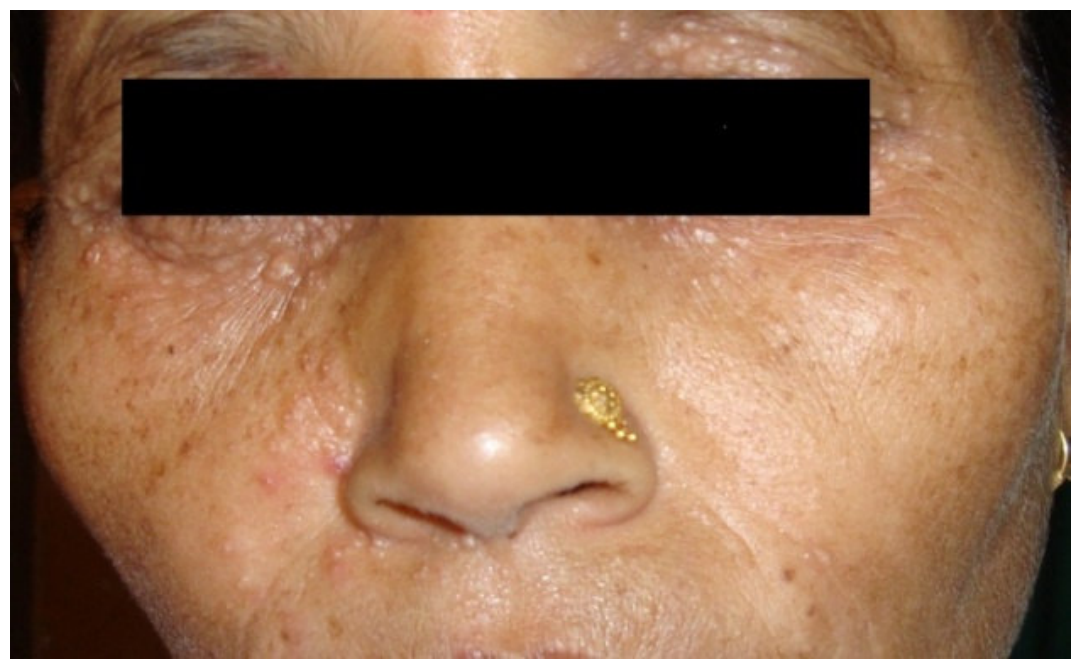

Figure-1: Syringoma

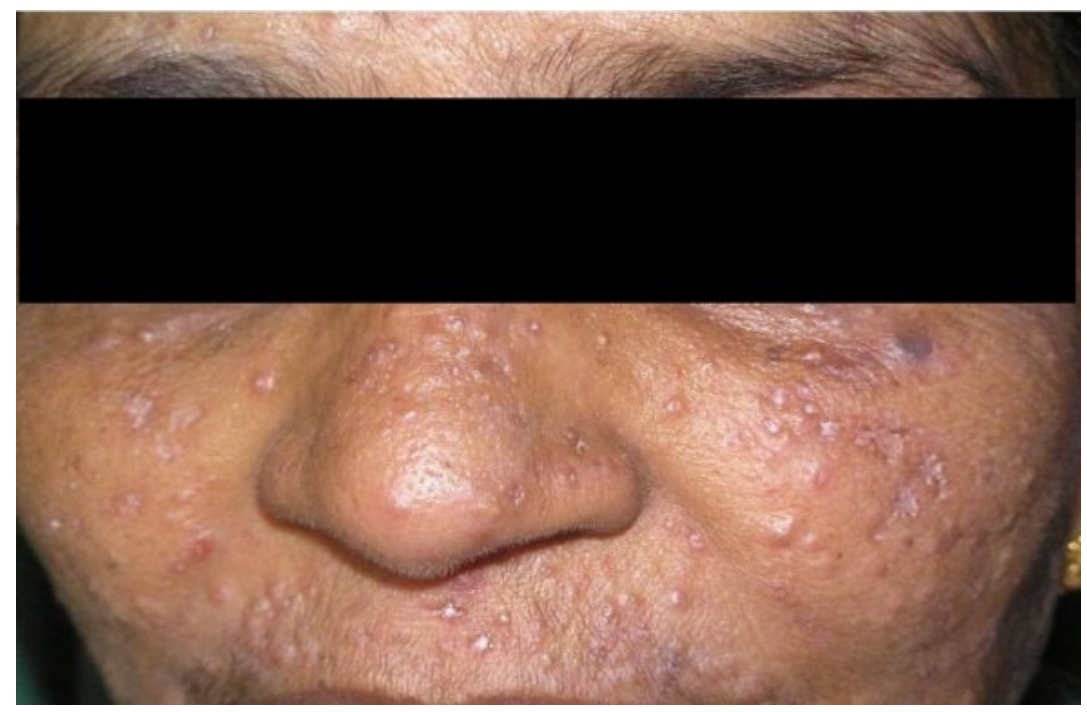

Figure-2: Trichoepithelioma

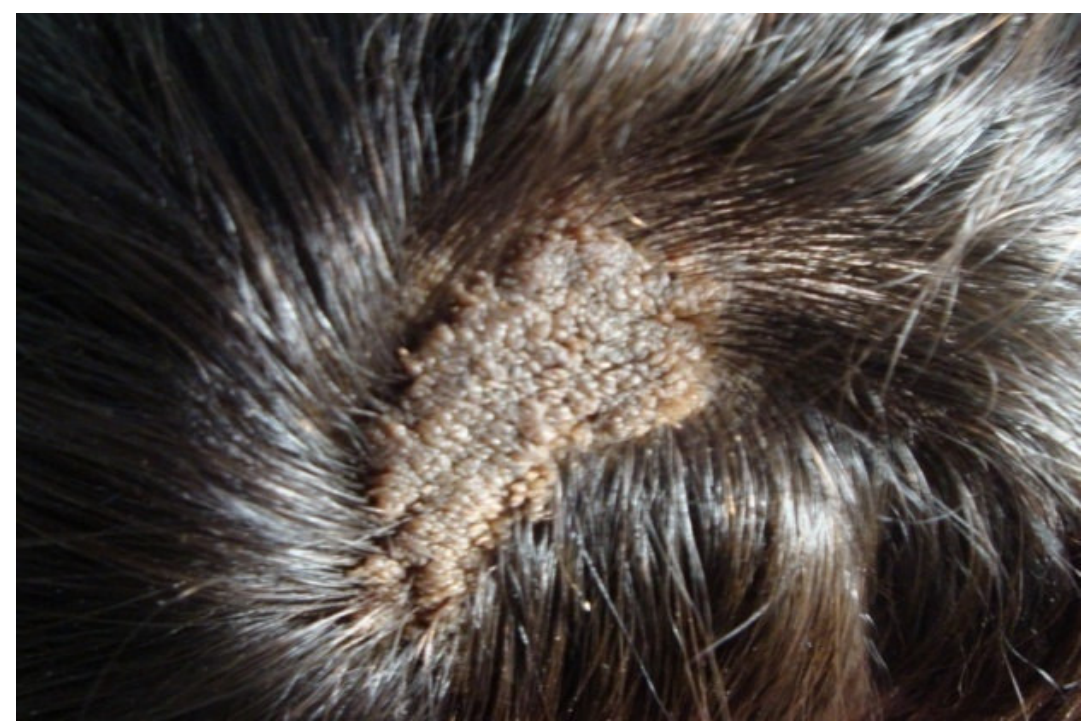

Figure-3: Nevus sebaceous 


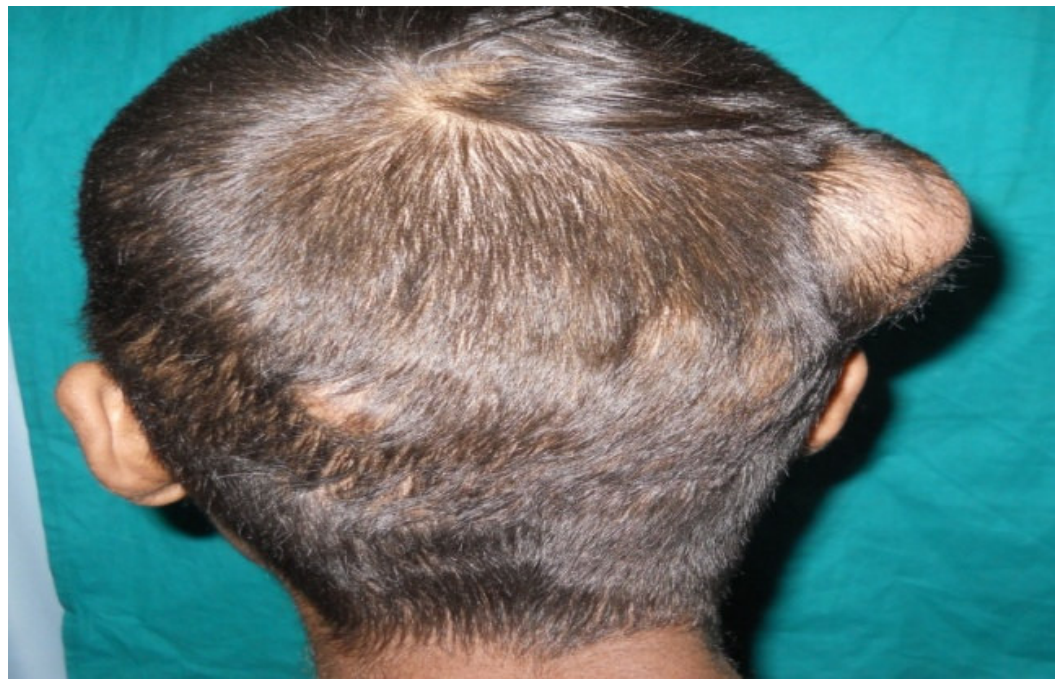

Figure-4: Cylindroma

In our study, nevus sebaceous (Figure No. 3), (5 patients $18.3 \%$ ) was present on its commonest site (scalp) with classical presentation as raised light brown plaque with velvety surface and histopathologically showed hyperkeratosis, acanthosis, epithelial papillomatosis of the epidermis, and defective sebaceous glands, whereas in a study done by Samaila M in Nigeria in 2008, nevus sebaceous were seen in $6(11.5 \%)$ of the total study subjects [5].

Other ATs like cylindroma (Figure No 4), hidrocystoma and eccrine spiradenoma were present with their usual clinichistopathological findings. In our study, on the basis of differentiation of tumors, sweat gland tumors were the most common appendageal tumors $(79.8 \%)$ followed by follicular tumors (11.6\%) and sebaceous $(8.3 \%)$, whereas in other study done by Jindal U \& Patel R in 2012, the most common tumors belonged to sweat gland differentiation $(52 \%)$, followed by hair follicle

(44\%). Only one case (4\%) belonged to sebaceous gland differentiation [6].

In our study, we found that all the ATs were of benign nature and no malignant tumour was found but Jindal $U$ and Patel R reported that most of ATs were benign (90\%) except one malignant tumor (4\%) of sweat gland (sweat gland carcinoma) in their study [6].

The majority of ATs were seen in age group of 20-40 years which is in contrast to other studies showing their onset at young age. This could be because of delay in seeking treatment due to benign nature of disease [6].

In our study, face was the commonest site of predilection (40 patients/66.6\%) followed by scalp (12 patients/20\%). Similar findings were observed in other studies $[5,7]$.
The early recognition of some ATs is also important as they might be markers of some syndromes associated with internal malignancy, like Cowden' syndrome. Syringoma and trichoepithelioma may be treated with diathermy, while surgical excision is the treatment of choice for other ATs [8].

\section{Conclusion}

Appendageal tumors are relatively uncommon and constitute very small proportion of dermatological disorders. Though clinical appearance might help in diagnosing common appendageal tumors but histological examination remains the gold standard method in diagnosing clinically suspicious lesions.

An effort should be made to examine patients properly to look for such skin tumors so as to increase the rate of diagnosis and therapeutic intervention. By doing same, we can raise the awareness of this group of skin tumors as this is one of the most extensively classified but highly neglected in routine clinical practice.

\section{Funding: Nil}

Conflict of interest: Nil

Permission from IRB: Yes

\section{References}

1. Gayathri Sri S, Alavandar E, S. Kumar A. An analysis of skin appendageal tumors in south India. Journal of Evolution of Medical and Dental Sciences. 2012; 1(6): 90712.

2. Mackie RM, Calonje E. Tumors of the Skin Appendages. In: Burns T, Breathnach S, Cox N, Griffiths C, editors. 
Rook's Textbook of Dermatology. $7^{\text {th }}$ edition. London: Blackwell Publishing Company; 2004. p. 1-34

3. Stantaylor R, Perone JB, Kaddu S, Kerl H. Appendage Tumors and Hamartomas of the Skin. In: Wolff K, Goldsmith L, Katz S, Gilchrest BA, Paller AS, Leffell DJ, ed. Fitzpatrick's Dermatology in General Medicine. $7^{\text {th }}$ edition. New York: McGraw Hill; 2008. p. 1068-87.

4. Saha A, Das NK, Gharami RC, Chowdhury SN, Datta PK. A clinico-histopathological study of appendageal skin tumors, affecting head and neck region in patients attending the dermatology OPD of a tertiary care centre in Eastern India. Indian J Dermatology 2011; 56:33-6.
5. Samaila M. Adnexal skin tumors in Zaria, Nigeria. Ann Afr Med 2008; 7:6-10.

6. Jindal U, Patel R. Study of adnexal tumors of the skin: a three year study of 25 cases. The internet journal of pathology. 2012; 13; 3 .

7. Sharma A, Deepak G. Paricharak, Nigam JS, Rewri S, Soni PB, Omhare A, Sekar P. Histopathological study of skin adnexal tumours-Institutional study in south India. Journal of Skin Cancer. 2014;1:1-4.

8. Nair PS. A clinicopathologic study of skin appendageal tumors. Indian J Dermatol Venereol Leprol 2008;74:550

\section{How to cite this article?}

Rathoriya SG, Soni SSL, Sinha U, Chanchlani R. Epidemiological study of Appendageal skin tumors in tertiary health centre in central India. Int J Med Res Rev 2014;2(2):86-91. doi:10.17511/ijmrr.2014.i02.03 